\title{
Detraining from total body exercise ergometry in individuals with spinal cord injury
}

\author{
Alfred Burke Gurney, Robert A Robergs, J Aisenbrey, JC Cordova and L McClanahan \\ Physical Therapy Division, University of New Mexico, HSSB 204 B, Albuquerque, New Mexico 87131-5661
}

\begin{abstract}
This study was designed to investigate the effects of detraining that occurred during an 8 week period of muscular inactivity following a 12 week training program of artificial computerized functional electrical stimulation cycle ergometry (CFES LE) and arm ergometry. Six spinal cord injured male individuals were followed through an 8 week detraining period that was preceded by a 12 week exercise program including CFES LE and arm ergometry. Maximal graded exercise tests were completed and measurements of peak oxygen consumption $\left(\mathrm{VO}_{2}\right)$, heart rate $(\mathrm{HR})$, ventilation $\left(\mathrm{V}_{\mathrm{E}}\right)$ workload, and creatine kinase were taken. Testing occurred at initial training (0T), after 12 weeks of training (12T), and after 8 weeks of detraining (DT). After the training program, peak $\mathrm{VO}_{2}$ increased significantly from $0.562 \pm 0.126$ (0T) to $1.021 \pm 0.247 \mathrm{l} / \mathrm{min}(12 \mathrm{~T}, P<0.05)$. After DT, peak $\mathrm{VO}_{2}$ decreased to $0.791 \neq 0.216 \mathrm{1} / \mathrm{min}$, which was lower than 12T $(P<0.05)$, yet higher than 0T $(P<0.05)$. After DT, peak workoad had decreased from $0.675 \pm 0.203(12 \mathrm{~T})$ to $0.32 \pm 0.203 \mathrm{kp}(P<0.05)$, which was not different than 0T. Creatine kinase levels were significantly lower both at 12T and DT compared to OT $(P<0.05)$. In addition, this training program induced linear increases in both $\mathrm{VO}_{2}$ and $\mathrm{HR}$ with workload, which were retained after DT. These increases did not reach statistical significance, however. No apparent relationship existed between these values at baseline. There were no significant differences in submaximal or peak HR of $V_{E}$ between the three testing periods. The results indicate that both peripheral muscular adaptations and central distribution adaptations in SCI individuals are partially maintained following 8 weeks of DT from CFES LE and arm ergometry.
\end{abstract}

Keywords: spinal cord; detraining; CFES LE

\section{Introduction}

Maintaining high levels of fitness may be more important for spinal cord injured (SCI) individuals than able-bodied individuals $(\mathrm{AB})$. For example, there is a significant influence of fitness status upon functional ability in SCI individuals ${ }^{1}$ and a short period of physical deconditioning in the SCI individual can significantly decrease quality of life, ultimately resulting in a state of complete dependency. ${ }^{2}$

Several methods have been shown to be efficacious in maintaining and improving fitness levels in SCI individuals. In 1984 Petrofsky et al $^{3}$ introduced an ergometer for use by SCI individuals that provided computerized functional electrical stimulation (CFES) to the legs. The stimulation consisted of a user controlled intensity to induce a sequence of coordinated contractions of the hamstrings, quadriceps, and gluteal muscle groups, enabling the SCI to perform leg ergometry (CFES LE).

Results of CFES LE training studies indicate that CFES LE affects several fitness parameters including increasing peak $\mathrm{VO}_{2}$ increasing peak workload,

Correspondence: A Burke Gurney increasing power output (PO), increasing peak heart rate, increasing stroke volume (SV), increasing cardiac output $(\mathrm{CO})$, increasing arteriovenous oxygen difference ( $\mathrm{a}-\mathrm{VO}_{2}$ diff), increasing peak ventilation, reducing total peripheral resistance (TPR), and reducing submaximal respiratory exchange ratio (RER). ${ }^{4-12}$ CFES has also been shown to affect heart tissue by increasing interventricular septal and posterior wall thickness as well as increasing left ventricular mass. ${ }^{13}$ In addition, CFES LE has been shown to decrease the rate of bone density loss in the tibia. ${ }^{14}$ Hybrid exercise (HE) involving CFES LE in conjunction with arm ergometry (AE) has also been shown to have endocrinological effects including lowering resting blood catecholamine (CAT) levels as well as increasing acute CAT levels. ${ }^{15}$

There is a large body of research regarding detraining of $\mathrm{AB}$ persons (see discussion), however, no study to date has evaluated the magnitude of detraining in SCI persons. This study was designed to measure peak oxygen consumption $\left(\mathrm{VO}_{2}\right)$, heart rate $(\mathrm{HR})$, ventilation $\left(\mathrm{V}_{\mathrm{E}}\right)$, maximal CFES LE workload (WL), and serum creatine kinase (CK) responses to 8 weeks of detraining from a previous 12 week CFES LE and arm ergometry training program. 
CFES LE has several limitations including cost and difficulty of application. Based on these limitations, it would be helpful to know what the minimum frequency of application of CFES LE would be to maintain some of the benefits. Finding the rate of detraining of SCI individuals from CFES LE and arm ergometry training could help minimize expense and inconvenience to the SCI individual during training.

The purpose of this study is to examine the rate of detraining that the individual with SCI undergoes in an 8 week course of detraining, both at the muscle tissue level via $\mathrm{CK}$ and $\mathrm{WL}$, and the cardiovascular level via $\mathrm{VO}_{2}, \mathrm{HR}$ and $\mathrm{V}_{\mathrm{E}}$. The rate of detraining of muscle tissue might give some insight into how frequently a spinal cord individual needs to train using a CFES LE device, the rate of detraining at the cardiovascular level might give insight into the specific mechanisms involved in detraining and how similar they are to able-bodied individuals.

A subset of the data used in this study was shared with previously published material. ${ }^{6}$ The original study involved training of the SCI individual and did not deal with detraining.

\section{Methods}

\section{Subjects}

Six adult male volunteers (ages 23-41) with spinal cord injuries resulting in paraplegia $(\mathrm{T} 4-10, n=4)$ and quadriplegia $(\mathrm{C} 4-7, n=2)$ who were eligible for CFES LE and voluntary AE were used for this study. The subjects had no prior experience in CFES LE training.

Prior to implementation of testing and treatment, each subject signed an informed consent approved by the University Medical School Human Research Review Committee. In accordance with the guidelines for CFES training recommended by Therapeutic Technologies Incorporated, each subject participated in pre-treatment screening tests. These tests included: a general physical exam, X-rays of the lower extremeties including anterior, posterior and lateral roentgenograms of the hips, knees, ankles, femurs and tibias to screen for the presence of fractures or severe osteoporosis; a physical therapy evaluation to assess joint range of motion, level of spasticity, spinal reflexes, and residual sensory and motor function; and a comprehensive hematology test to determine whether blood markers for liver and kidney function were within normal limits. Of a potential subject pool of over $50 \mathrm{SCI}$ volunteers, eight met the criteria for CFES LE, and of these, six subjects completed the entire training and detraining sequence. The major reasons for non-inclusion were the presence of enough lower extremity sensation to render the electrical stimulation intolerable and the absence of either quadriceps and ankle jerk reflexes. The difficulties in recruiting subjects for this research prevented a larger sample size and a suitable control group.
CFES LE was performed using the ERGYS 1 system (Therapeutic Technologies, Inc. Tampa FL). The ERGYS 1 system applies a graded increase in current to the skin surfaces over the quadriceps, hamstrings, and gluteal muscles. Maximal current output is 132 mamps. During incremental CFES LE, the ergometer is computer programmed to increase resistance in increments of $1 / 8 \mathrm{kp}$. When subjects can no longer maintain a pedaling frequency of $35 \mathrm{rpms}$, the system is programmed to terminate current output. To ease the transition between rest and exercise, and exercise and recovery, a technician assisted the subject by manually turning the crankshaft.

\section{Testing procedures}

Procedures used during testing are described in detail by Krauss et $a l^{6}{ }^{6}$ In summary, subjects first completed a CFES LE test for the evaluation of blood serum CK. Prior to the test, blood was drawn from an antecubital vein (Table 2, pre 0T), and the subjects were then positioned and connected to the CFES ergometer. Subjects completed CFES LE with zero resistance and cycled at a cadence of $50 \mathrm{rpm}$. The test was halted when the cadence declined to $35 \mathrm{rpm}$ or $30 \mathrm{~min}$ had elapsed. Failure to maintain a pedaling frequency of $35 \mathrm{rpm}$ automatically terminated delivery of CFES signals to the subject. After this session, the subjects did not exercise for the next $72 \mathrm{~h}$. Subjects returned to the lab 24, 48 and $72 \mathrm{~h}$ after this first test for additional venous blood sampling (Table 2, 0T). The highest value of the three samples was taken and designated the peak creatine kinase (pk CK) as it has been shown by Hertobagyi and Denahan ${ }^{16}$ that pk CK may not occur for $24-48 \mathrm{~h}$.

At least $72 \mathrm{~h}$ following the first CFES LE test, subjects completed an intermittent graded CFES LE test to determine peak oxygen consumption $\left(\mathrm{VO}_{2}\right)$, heart rate (HR) and workload in kiloponds $(\mathrm{kp})$ response to CFES LE.

The test protocol consisted of a 1 min warm up with assisted pedaling. The subject then received stimuli and pedaled unassisted without resistance for $3 \mathrm{~min}$. After each $3 \mathrm{~min}$ exercise period there was a 2 min cool down period with assisted pedaling and a $1 \mathrm{~min}$ warm-up period with assisted pedaling. There were incremental increases in resistance of $1 / 8 \mathrm{kp}$ each three minute stage. This protocol continued with $1 /$ $8 \mathrm{kp}$ incremental increases until the subject was not able to pedal against the prescribed resistance at a cadence greater than $35 \mathrm{rpm}$.

During each test, the subject's HR was monitored continuously by telemetry (Computer Instruments Corporation, Hempstead, NY, model 8799). During incremental exercise tests, a Gould $9000^{\mathrm{TM}}$ Cardiopulmonary Exercise System was used to monitor cardiorespiratory responses, as previously described ${ }^{6}$ Measurements of oxygen consumption $\left(\mathrm{VO}_{2} 1 \mathrm{~min}^{-1}\right)$ and respiratory exchange ratio (RER) were determined from averaged data during the last minute of each 
stage. The incremental exercise tests were completed at baseline (0T), week 12 (12T), and again following 8 weeks of detraining (DT). CK values were taken 24,48 and $72 \mathrm{~h}$ after each of these tests from which pk CK values were calculated. In addition, a baseline CK immediately preceding CFES LE at eight weeks of detraining was taken (pre-DT) (Table 2).

\section{Training procedures}

The training program consisted of three phases (Figure 1). Procedures used during training are described in detail by Krauss et al. ${ }^{6}$

Phase I consisted of CFES LE training for 6 weeks, three times a week. This phase included a intermittent training protocol where each subject pedaled with stimulation at increasing time intervals and with increasing resistance. Time intervals were increased as each individual became stronger and responded with longer intervals to the stimuli.

Phase II consisted of a 6 week training period of HE three times a week. The leg training program described in Phase I was continued. In addition, the subject pedaled the Monark arm ergometer during CFES LE.

Phase III consisted of an 8 week detraining period in which the subjects did not participate in either the CFES LE or the HE. Those subjects who were involved in competitive wheelchair athletics were told to refrain from any training activities during this time. Procedures used during training with CFES LE are described in detail by Krauss et al. ${ }^{6}$

\begin{tabular}{||c|c|c||}
\hline $\begin{array}{c}\text { Phase I - 3x/wk for } \\
6 \mathrm{wks}\end{array}$ & $\begin{array}{c}\text { Phase II - 3x/wk for } \\
6 \mathrm{wks}\end{array}$ & $\begin{array}{c}\text { Phase III - } \\
8 \mathrm{wks}\end{array}$ \\
\hline $\begin{array}{c}\text { Computerized } \\
\text { electrical } \\
\text { stimulation leg } \\
\text { ergometry (CFES LE) } \\
\text { alone }\end{array}$ & $\begin{array}{c}\text { Hybrid Ergometry (HE) } \\
\text { (CFES LE and arm }\end{array}$ & $\begin{array}{c}\text { Detraining - no } \\
\text { ergometric } \\
\text { ergometry in }\end{array}$ \\
\hline
\end{tabular}

Figure 1 Training program

\section{Analytical procedures}

Blood samples were left to clot at room temperature, centrifuged at $4^{\circ} \mathrm{C}$, and the serum was removed and frozen at $-14^{\circ} \mathrm{C}$ until subsequent analyses. Serum samples were assayed in duplicate for CK using a commercial kit (Sigma Diagnostics ${ }^{\mathrm{TM}}$ \#47-UV) based on the Szasz procedure. CK activity was measured at $340 \mathrm{~nm}$ using a Spectronic 401 spectrophotometer (Milton Roy ${ }^{\text {TM }}$ ) programmed to calculate CK activity expressed as $\Delta$ absorbance $\mathrm{min}^{-1}$ from a $2 \mathrm{~min}$ sampling time. CK absorbance values were then converted to Ktals $1^{-1}$.

\section{Statistical analyses}

Each of the peak $\mathrm{VO}_{2}, \mathrm{RER}, \mathrm{V}_{\mathrm{E}}$, workload (WL) and submaximal and maximal $\mathrm{HR}$ data were analyzed by one-way ANOVA with repeated measures (Statview ${ }^{\mathrm{TM}}$, Abacus Concepts, Inc., Berkeley). The peak CK responses data did not meet ANOVA requirements of homogeneity of variance, and were converted to their natural logarithm (In pk CK) and then analyzed by one-way ANOVA with repeated measures. Mean pairwise comparisons were evaluated by the Scheffé test.

The relationship between $\mathrm{HR}$ and $\mathrm{VO}_{2}$ was assessed for each subject by simple linear regression (GraphPad Prism, San Diego). The residual errors around each line of best fit were assessed from standard errors of the estimate, and the slope of the line of best fit was used to reflect the physiological 'normality' of the HR$\mathrm{VO}_{2}$ response to CFES-LE. $T$-tests were completed comparing the standard error of estimate and the regression slope of $12 \mathrm{~T}$ and DT. Statistical significance was accepted at $P<0.05$.

\section{Results}

Only five of the original eight subjects returned for the evaluation of detraining responses. One additional subject who was the original pilot for the research and not included in the original eight ${ }^{6}$ was added into the analyses to give the total of six subjects. The descriptive characteristics of the subjects are presented in Table 1.

CFES LE induced higher serum CK activity after baseline testing (pre 0T versus 0T, Table 2). Analyses of the In pk CK data revealed that the CK response

Table 1 The descriptive and physical characteristics of the subjects

\begin{tabular}{lccccc}
\hline Subject $\#$ & Age & Gender & Level of Injury & Years post Injury & Spasticity level \\
\hline 1 & 25 & M & C4 & 5 & moderate \\
2 & 41 & M & T4 & 24 & moderate \\
3 & 35 & M & T4 & 15 & moderate \\
4 & 23 & M & T10 & 5 & moderate \\
5 & 29 & M & T8 & 9 & moderate \\
6 & 28 & M & & 6 & severe \\
\hline
\end{tabular}


was significantly less at $12 \mathrm{~T}$ and DT compared to $0 \mathrm{~T}$, and significantly higher than baseline (Figure 2).

Subjects increased their ability to exercise by CFES LE, as indicated by the progression of $\mathrm{pk}$ WL from baseline to week 12 (Figure 3). The 8 week DT period significantly decreased pk WL to a value similar to baseline testing, however, the pk $\mathrm{VO}_{2}$ was still significantly higher than baseline (Figure 4). Despite the changes in pk WL and pk $\mathrm{VO}_{2}$, the submaximal and peak HR responses to CFES LE during the training and detraining were not significantly different. The $\mathrm{pk} H \mathrm{HR}$ and $\mathrm{pk} \mathrm{V}_{\mathrm{E}}$ data are presented in Table 2 .

There was no difference between the residual errors or slopes from linear regression for the $\mathrm{HR}-\mathrm{VO}_{2}$

Table 2 The absolute peak serum creatine kinase activity (pk CK, Ktals $\mathrm{L}^{-1}$ ), peak heart rate $(\mathrm{HR}, \mathrm{bpm}$ ) and peak ventilation $\left(\mathrm{V}_{\mathrm{E}} \mathrm{L} \mathrm{min}^{-1}\right)$ data $($ mean $\pm \mathrm{SE})$

\begin{tabular}{lccl}
\hline & $p k C K$ & $p k H R$ & $p k V_{E}$ \\
\hline pre 0T & $32.0 \pm 18.88$ & & \\
0T & $1371.6 \pm 956.12$ & $77.7 \pm 25.7$ & $21.9 \pm 5.8$ \\
12T & $59.0 \pm 18.7$ & $104.3 \pm 37.15$ & $30.8 \pm 5.1^{*}$ \\
pre-DT & $137.0 \pm 24.5$ & & \\
DT & $167.0 \pm 71.1$ & $91.5 \pm 21.5$ & $25.4 \pm 8.05$ \\
\hline
\end{tabular}

*Significantly different from $0 \mathrm{~T}, P<0.05$

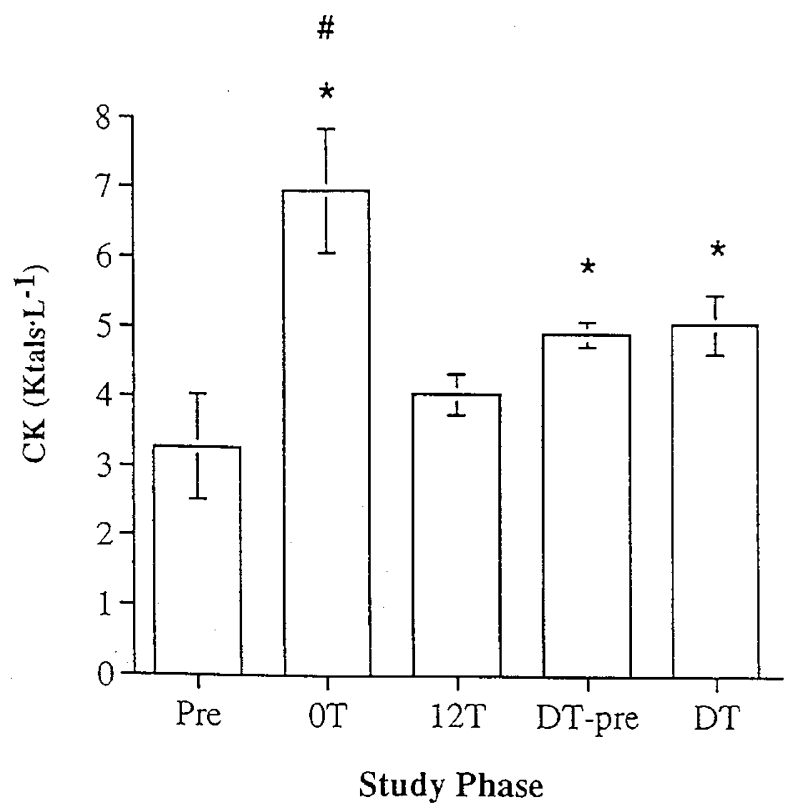

Figure 2 The changes in the natural $\log$ of peak serum creatine kinase activity (In pk CK) during training and detraining. The In pk CK was significantly higher after the CFES LE test at 0T than all other values (\#). In addition, In pk CK at DT-pre and DT was significantly higher than pre-0T response between $12 \mathrm{~T}$ and DT. Nevertheless, upon visual inspection of the data, the relationship between $\mathrm{HR}$ and $\mathrm{VO}_{2}$ during CFES LE appeared to change in response to the training (Figure 5).

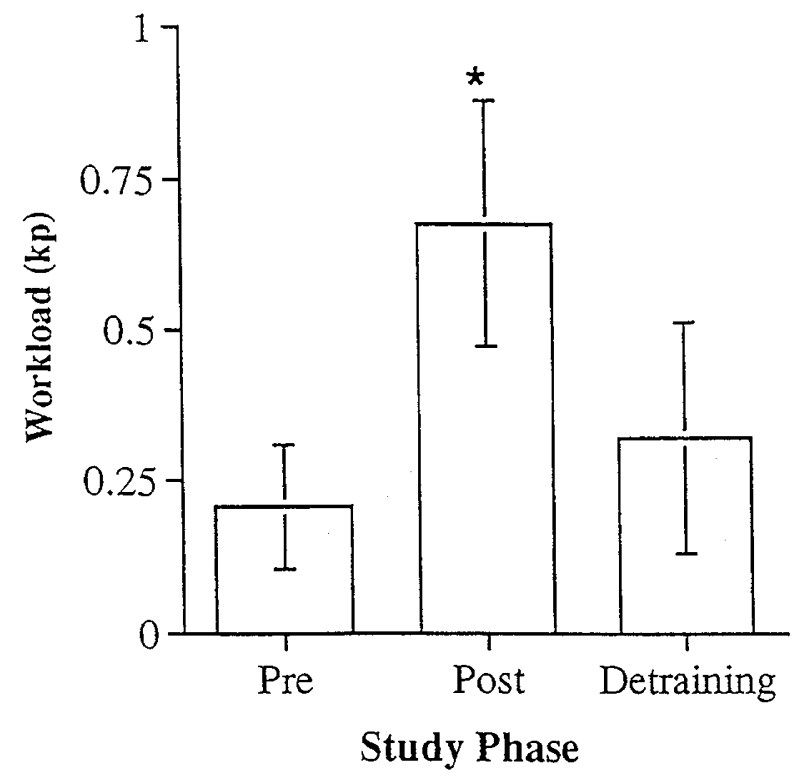

Figure 3 The differences in workload $(\mathrm{kp})$ between pretraining (pre), 12 weeks of training (post), and 8 weeks of detraining. The workload after training was significantly different from both pre-training and detraining $(*)$

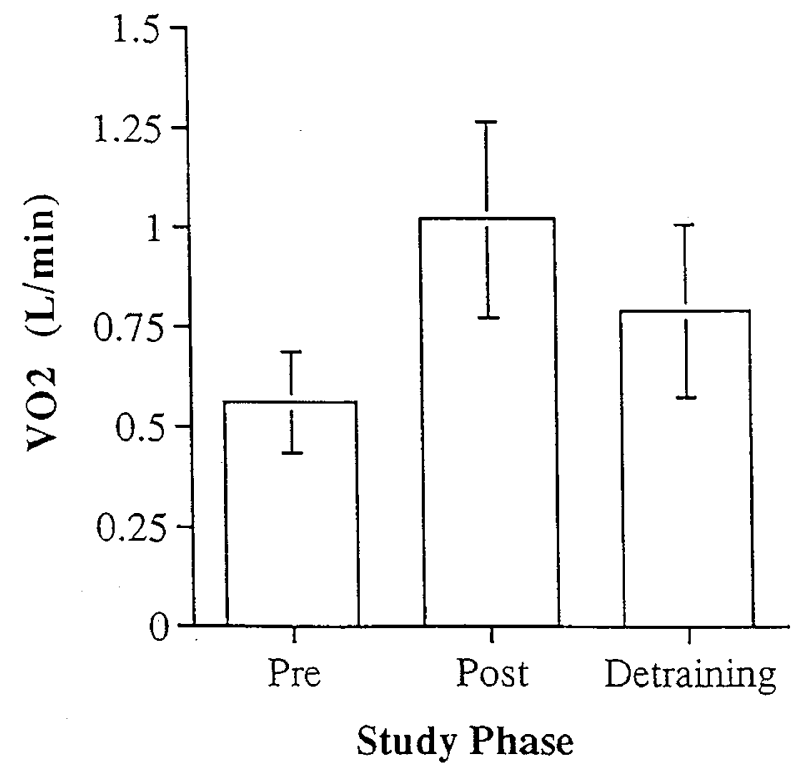

Figure 4 The change in peak oxygen consumption $\left(\mathrm{pkVO}_{2}\right)$ during training and 8 weeks of detraining. $\mathrm{Pk} \mathrm{VO}_{2}$ values were significantly different at each training phase 
There appeared to be no pattern to the response to CFES LE based on the level of injury, or years since injury (Table 3).

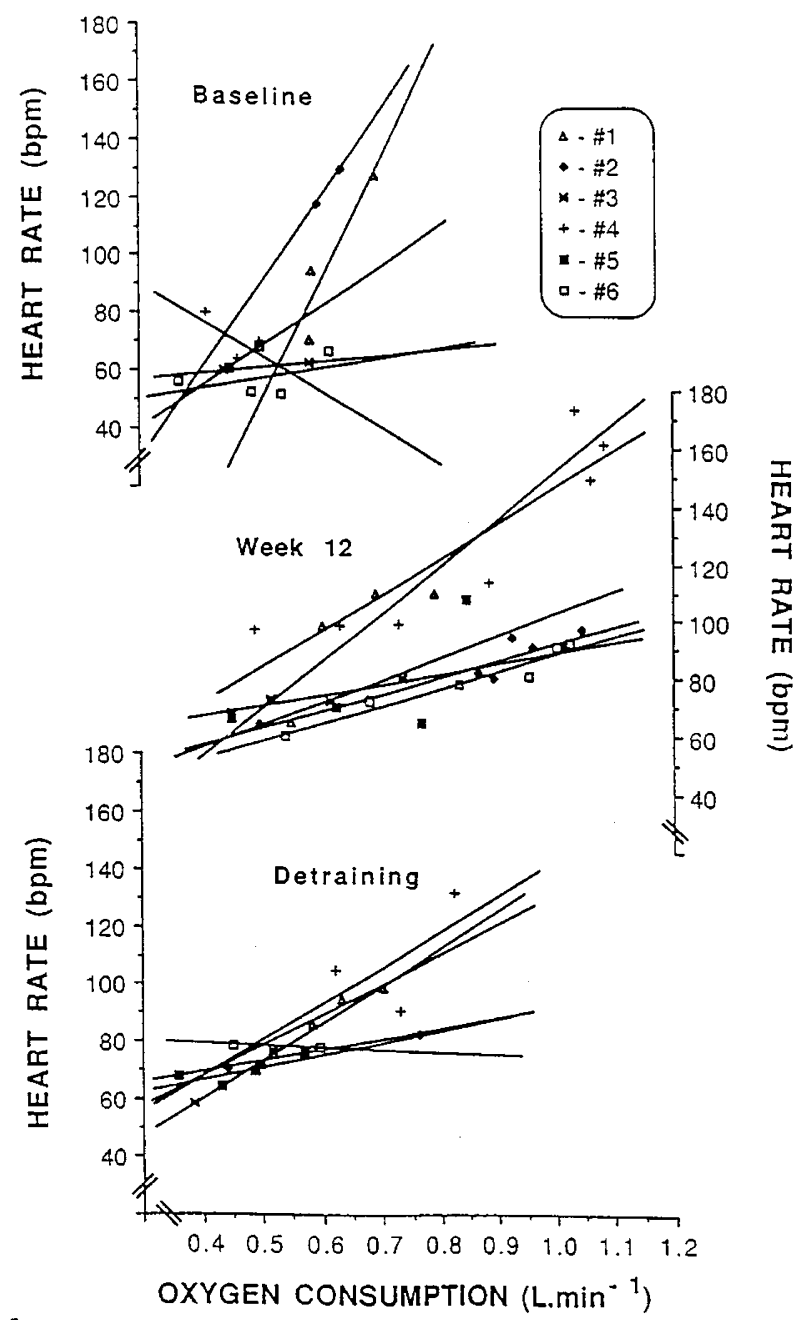

Figure 5 The relationships between increasing workload and heart rate and oxygen consumption $\left(\mathrm{VO}_{2}\right)$ at baseline, after 12 weeks of training, and after 8 weeks of detraining. Subjects \#'s correspond to Table 1

\section{Discussion}

The training protocol resulted in an increase in exercise tolerance to CFES LE. Subjects were able to perform CFES LE to an increased peak WL and peak $\mathrm{VO}_{2}$. Blood CK activity decreased with training despite these increased WL and associated increased durations of stimulation. Despite 8 weeks of DT, the blood CK activity resulting from CFES LE remained significantly lower than pre-training. In addition, peak $\mathrm{VO}_{2}$ levels during CFES LE remained significantly higher than pre-training levels. These results indicate a retention of benefits from the training protocol for at least 8 weeks.

The association of the training benefits and CFES LE alone is complicated by the inclusion of arm ergometry during phase II of the protocol. The use of HT in phase II of the training protocol may have caused a carryover of the upper extremity component on cardiovascular fitness levels. It has been shown in AB populations that improvements in LE tested peak $\mathrm{VO}_{2}$ occur while training predominantly the $\mathrm{UE}^{17}$ and it is likely the SCI population would do so as well. However, as the testing condition at each phase was only CFES LE, the CK data pertain only to the artificially stimulated muscle (ie LE muscle). Regardless of the peripheral metabolic consequences of CFES LE training, the ability for paralyzed muscle to retain contractile and metabolic responses to artificial stimulation after 8 weeks of inactivity is remarkable.

The results of this investigation are the first to document the retention of muscular endurance of paralyzed muscle and cardiovascular adaptations following a training program.

Following spinal cord injury resulting in paraplegia or quadriplegia, the denervated skeletal muscle alters in morphology and function due to fiber atrophy, and a slow-to-fast shift in myofibrillar properties occurs. ${ }^{9,18}$ Artificial stimulation of paralyzed muscle provides gross synchronous contractions without the fine neural control of the alpha and gamma reflex circuitry from the muscle spindles that exists in voluntary contractions. Although CFES LE induced muscle damage is poorly researched in SCI individuals, ${ }^{12}$ it would appear that a greater potential for muscle damage exists during CFES LE than in voluntary muscle contraction, possibly due to the

Table 3 Individual subject exercise workloads* during incremental CFES LE at different training phases

\begin{tabular}{|c|c|c|c|}
\hline $\begin{array}{l}\text { Subject (injury level); } \\
\text { Years post injury }\end{array}$ & $\begin{array}{l}\text { Baseline } \\
(\mathrm{kg})\end{array}$ & $\underset{(\mathrm{kg})}{\text { Training }}(12 T)$ & $\underset{(\mathrm{kg})}{\operatorname{Detraining}}(D T)$ \\
\hline 1 (C4); 5 years & 0.250 & 0.375 & 0.250 \\
\hline 2 (T4); 24 years & 0.125 & 0.875 & 0.250 \\
\hline 3 (C7); 15 years & 0.125 & 0.375 & 0.250 \\
\hline 4 (T4); 5 years & 0.250 & 0.750 & 0.250 \\
\hline 5 (T10); 9 years & 0.125 & 0.375 & 0.375 \\
\hline 6 (T8); 6 years & 0.375 & 0.625 & 0.125 \\
\hline
\end{tabular}

*Workload increments were $0.0125 \mathrm{~kg}$ 
synchronicity of recruitment and subsequent inability for motor units to continuously alternate activity and thus share the work. Our CK data (Table 2, Figure 1) indicate that acute exposure to CFES LE induces muscle damage. The increase in serum CK activity following CFES LE in our subjects is not large compared to values in excess of $10000 \mathrm{Ktals} . \mathrm{L}^{-1}$ in the $\mathrm{AB}$ population following either marathon running or damage caused by eccentric muscle contractions. ${ }^{16,19}$ However, the SCI individual has a reduced muscle mass, and the duration of the combined exercise duration of the initial test was less than $10 \mathrm{~min}$ and did not include an eccentric component. Given these considerations the CK responses of the SCI individuals were relatively large. Compared to the first CFES LE test, the reduced CK response to more prolonged CFES LE after training, and even after DT, indicated that the muscle may have adapted and retained tolerance to artificial electrical stimulation for a considerable period of time.

Martin et $a l^{9}$ demonstrated that 24 weeks of unloaded electrical stimulation of the tibialis anterior of human SCI individuals caused significant increases in the proportion of type I fibers, the mitochondrial enzymatic activity of both type I and II fibers, and the contractile endurance of the muscle. A trend existed for an increase in muscle capillarization. Despite the shorter training duration of our study compared to Martin et $a l^{9}(12-24$ weeks), it is reasonable to assume that the electrically induced contractions of the larger muscle groups of the upper leg and buttocks against progressively increased resistance should have also induced increases in muscle oxidative capacities. These adaptations would help explain the dramatic improvements in muscular endurance and peak oxygen consumption noted in our subjects (Figure 3 and 4 ).

For the SCI individual, discontinuation from CFES LE training is probably the most severe form of DT possible for the lower limbs. Without artificial electrical stimulation, the only contractile activity that can occur in paralyzed muscle is from spasticity. Although data of altered spasticity was not included in the study, observation and subject feedback indicated that spasticity did not increase during the training period, or during DT. As the 8 weeks of DT did not return muscle endurance or peak oxygen consumption to pre-training values, some of the improvements in muscle oxidative capacity could have been retained. This is an interesting finding compared to the response of normal subjects to similar duration detraining periods. As there is no other research of detraining from CFES LE, we can only compare our results to detraining or limb immobilization in $\mathrm{AB}$ individuals.

MacDougall et $a l^{20}$ evaluated muscle fiber morphology of the triceps brachii following 6 weeks of immobilization in an elbow cast. Immobilization decreased both slow and fast twitch fiber areas, without a change in fiber type proportion. Costill et $a l^{21}$ measured quadriceps strength and endurance, and the oxidative capacity of biopsy specimens from the vastus lateralis muscle before and after knee surgery. After 2 weeks the cast immobilization, the surgically treated leg had a $26-42 \%$ decrease in succinate dehydrogenase (SDH) activity, indicating reduced mitochondrial density. Similar results were obtained by Sargeant et $a l^{21}$, but for a longer duration of cast immobilization ( $7-30$ weeks), and indicated that in immobilization models with intact neural innervation, no slow-to-fast myofibrillar changes occur in the atrophied fibers.

The rapidity of the muscle detraining response is even seen in DT from exercise in the AB. Additional research by Costill et $a l^{23}$ using collegiate swimmers indicated that the respiratory capacity of the posterior deltoid decreased $50 \%$ within the first week of detraining, yet did not decrease further during the remaining 4 weeks of the study. Similar reductions in muscle respiratory capacity have also been obtained from DT from running ${ }^{24}$ and cycle ergometry ${ }^{25}$ in the AB. Interestingly, Klausen et al first trained subjects by cycle ergometry for 8 weeks, and then DT the subjects for 8 weeks. Despite dramatic reductions in muscle mitochondrial enzyme activities to pre-training levels, capillarization adaptations were retained during DT and $\mathrm{VO}_{2}$ max still remained significantly higher than pre-training. It is apparent that the loss of respiratory potential in $\mathrm{AB}$ human skeletal muscle occurs rapidly during DT, yet does not necessarily indicate similar reductions of oxygen extraction and consumption capabilities.

Our data indicate that the low functional capacity of paralyzed muscle, even after electrical stimulation training, may not detrain as rapidly as the highly trained muscle of $\mathrm{AB}$ individuals. Muscle biopsy specimens would need to be obtained to conclusively support this fact, however, the retention of muscle contractile endurance and $\mathrm{VO}_{2}$ in our subjects is apparent.

An additional finding from our study is that the $\mathrm{HR}-\mathrm{VO}_{2}$ relationship during CFES LE was altered with training, and remained similar to the immediate post-training results after the DT. Although this relationship did not reach statistical significance, visual inspection shows a clear change in profiles (Figure 5). At baseline, no apparent relationship existed between increases in workload and the subject's $\mathrm{HR}$ and $\mathrm{VO}_{2}$. The limited number of participants in this study is the probable explanation for lack of statistical significance. The 12 weeks of training invoked a linear increase in both $\mathrm{VO}_{2}$ and HR with workload, which was retained after DT despite lowered CFES LE exercise endurance and pk $\mathrm{VO}_{2}$.

Bar-On et $a l^{26}$ reported that the $\mathrm{HR}-\mathrm{VO}_{2}$ relationship is linear for both paraplegic and quadriplegic subjects during arm ergometry. However, their data cannot be compared to $\mathrm{HR}-\mathrm{VO}_{2}$ changes during CFES LE by the SCI individual given the variable magnitude 
of peripheral pooling of blood and resulting impairment to venous return during upper body exercise in the SCI individual. ${ }^{27-32} \mathrm{In}$ fact, Irizawa et $a l^{33}$ demonstrated that, during arm crank exercise, the higher the level of the SCI lesion, the higher the HR at a given $\mathrm{VO}_{2}$. The $\mathrm{HR}$ response to increased $\mathrm{VO}_{2}$ during CFES LE was less dramatic in our subjects compared to the results of Bar-On et al, ${ }^{26}$ which confirm the difficulties in using HR as a measure of intensity during upper body exercise in the SCI individual. Our findings are meaningful in that the subjects demonstrated a relationship between exercise intensity induced by artificial stimulation and an increase in HR, that this relationship was more consistent after training, and was retained following DT. The retained relationship following DT might be explained by Bloomfield et $a l^{15}$ who found that training of SCI individuals resulted in increased CAT levels in response to acute exercise.

The minimal DT in the SCI individual may indicate that once a certain level of CFES LE training adaptation has occurred, additional training may not need to continue at the initial frequency and/or duration. If so, this would decrease cost of CFES LE to the SCI individual, and enable a larger number of SCI individuals to utilize this form of training.

The relatively low number of individuals (8) that were eligible for the study compared to the original participants (50) deserves some discussion. As stated before, the major reasons for non-eligibility was the presence of sensation in the lower extremities and absence of either quad or plantar flexor reflexes. A large number of potential participants were either incomplete spinal cord injured, which left them with some sensation, or had associated peripheral nerve injuries, most commonly lesions below the L1 level which resulted in loss of reflexes. The former group could not generally tolerate the magnitude of the electrical stimulation necessary to pedal the cycle ergometer, the latter were unresponsive to the wave forms of electricity used in the experiment. Areas of further research include finding the minimum frequency of CFES LE training to maintain training levels of peak $\mathrm{VO}_{2}$, peak work load, and LE muscular adaptations.

\section{Conclusions and recommendations}

Following 12 weeks of training by SCI individuals, an 8 week period of DT decreased peak $\mathrm{VO}_{2}$ by $23 \%$, decreased the peak workload during a progressive exercise test by $52 \%$, and blunted the serum CK activity responses to CFES LE. These results indicate that despite inactivity for 8 weeks, paralyzed muscle can partially retain previous adaptations to artificial electrical stimulation and muscle contraction, and cardiopulmonary endurance is maintained somewhat. The results also provide further proof of the effectiveness of CFES LE as part of a rehabilitation program for the SCI individual.

\section{References}

1 Noreau et al. Relationship of impairment and functional ability to habitual activity and fitness following spinal cord injury. Inter J Rehab Research 1993; 16: $265-275$.

2 Petrofsky JS, Phillips CA, Heaton HH, Glaser RM. Bicycle ergometer for paralyzed muscle. J Clin Engineering 1984; 9: $13-$ 18 .

3 Noreau L, Shepard RJ. Spinal cord injury, exercise and quality of life. Sports Medicine 1995; 20: 226-250.

4 Hooker SP et al. Peak and submaximal physiologic responses following electrical stimulation leg cycle ergometer training. $J$ Rehab Research Dev 1995; 32: 361 - 366.

5 Nash MS et al. Effects of electrically-stimulated exercise and passive motion on echocardiographically-derived wall motion and cardiodynamic function in tetraplegic persons. Paraplegia 1995; 33: 80-89.

6 Krauss JC et al. Cardiorespiratory effects of computerized functional electrical stimulation (CFES) and hybrid training in individuals with spinal cord injuries. Med Sci Sports Exerc 1993; 25: $1054-1061$.

7 Hooker SP et al. Physiologic effects of electrical stimulation leg exercise training in spinal cord injured persons. Arch of Physical Medicine Rehab 1992; 73: 470-476.

8 Goss F, McDermott A, Robertson J. Changes in peak oxygen uptake following computerized functional electrical stimulation in the spinal cord injured. Research Quarterly for Exercise and Sport 1992; 63: 76-79.

9 Martin TP, Stein RB, Hoeppner PH, Reid DC. Influence of electrical stimulation on the morphological and metabolic properties of paralyzed muscle. J Appl Physiol 1992; 72: 1401 1406.

10 Hooker SP et al. Physiologic responses to prolonged electrically stimulated leg-cycle exercise in spinal cord injured. Archives of Physical Medicine and Rehabilitation 1990; 71: 863-868.

11 Pollock SF et al. Aerobic training effects of electrically induced lower extremity exercises in spinal cord injured people. Archives of Physical Medicine and Rehabilitation 1989; 70: 214-219.

12 Ragnarsson KT. Physiologic effects of functional electrical stimulation-induced exercises in spinal cord-injured individuals. Clinical Orthopedics 1988; 233: 53-63.

13 Nash MS et al. Reversal of adaptive left ventricular atrophy following electrically-stimulated exercise training in human tetraplegics. Paraplegia 1991; 29: 590-599.

14 Hangartner TN, Rodgers MM, Glaser RM, Barre PS. Tibial bone density loss in spinal cord injured patients: effects of FES exercise. J Rehab Research Develop 1994; 31: 50-61.

15 Bloomfield SA, Jackson RD, Mysiw WJ. Catecholamine response to exercise and training in individuals with spinal cord injury. Med Science Sports Exer 1994; 26: 1213-1219.

16 Hortobagyi T, Denahan T. Variability in creatine kinase: Methodological, exercise, and clinically related factors. Int $J$ Sports Med 1989; 10: 69-80

17 McKenzie DC, Fox EL, Cohen K. Specificity of metabolic and circulatory responses to arm and leg training. Eur J Appl Physiol 1978; 39: $241-248$.

18 Ansved T, Larsson L. Effects of denervation on enzymehistochemical and morphological properties of the rat soleus muscle in relation to age. Acta Physiol Scand 1990; 139: 297-304.

19 Costill DL et al. Muscle glycogen resynthesis after eccentric exercise. J Appl Physiol 1990; 69: 46-50.

20 MacDougall JD et al. Effects of strength training and immobilization on human muscle fibers. Eur J Appl Physiol 1980; 43: $25-34$.

21 Costill DL, Fink WJ, Habansky AJ. Muscle rehabilitation after knee surgery. Physician and Sports Med 1977; 5: 71-74.

22 Sargeant AJ et al. Functional and structural changes after disuse of human muscle. Clin Sci Mol Med 1977; 52: 337-342.

23 Costill et al. Muscle changes during de-training. Swim Techn 1985; August-October: $15-18$ 
24 Moore RL et al. Effect of training/detraining on submaximal exercise responses in humans. J Appl Physiol 1987; 63: 17191724.

25 Klausen K, Anderson LB, Pelle I. Adaptive changes in work capacity, skeletal muscle capillarization and enzyme levels during training and detraining. Act Physiol Scand 1981; 113: 9-16.

26 Bar-On ZH, Nene AV. Relationship between heart rate and oxygen uptake in thoracic level patients. Paraplegia 1990; 28: $87-95$.

27 Arrowood JA, Mohanty PK, Thames MD. Cardiovascular problems in the spinal cord injured patient. Physical Medicine and Rehabilitation: State of the Art Reviews 1987; 1: 443-456.

28 Cowel LL, Squires WG, Raven PB. Benefits of exercise for the paraplegic: a brief view. Med Sci Sports and Exerc 1986; 18: 501 508.
29 Davis GM et al. Cardiovascular responses to arm cranking: FNSinduced leg exercise in paraplegics. J Appl Phys 1990; 69: 671677

30 Edwards BG, Marsolais EB. Metabolic responses to arm ergometry and functional neuromuscular stimulation. J Rehab Res Devel 1990; 27: 107-114.

31 Glaser RM. Exercise and locomotion for the spinal cord injured. Exercise and Sport Sciences Reviews 1985; 13: 263-303.

32 Glaser RM. Physiologic aspects of spinal cord injury and functional neuromuscular stimulation. Central Nervous System Trauma 1986; 3: 49-62.

33 Irizawa $\mathrm{M}$ et al. Relationship between heart rate and oxygen uptake during submaximal arm cranking in paraplegics and quadriplegics. Annals of Physiological Anthropology 1994; 13: 275 - 280 . 